\title{
Reliability-based Optimization Design of Mechanical Components with Truncated Normal Distributions
}

\author{
Xiangdong $\mathrm{He}^{1, \mathrm{a}}$, Xiaoyan $\mathrm{Hu}^{1, \mathrm{~b}}$, Wei Qi ${ }^{1, \mathrm{c}}$ \\ ${ }^{1}$ School of Mechanical Engineering \& Automation, Dalian Polytechnic University, Dalian 116034, \\ China \\ ahexd@dlpu.edu.cn, b1689680204@qq.com, cweiqi@dlpu.edu.cn
}

\begin{abstract}
Keywords: Mechanical components, Truncated normal distribution, Maximum entropy, Reliability-based optimization design

Abstract. In some engineering applications, the probability distributions of some random variables are truncated. Under the conditions, if traditional reliability-based optimization design is directly used, difficulties may arise and this treatment may result in large errors in reliability-based optimization design. In view of above mentioned problems, the paper is to present a new reliability-based optimization design method that solves the problems with truncated normal random variables. The numerical examples are investigated to demonstrate the effectiveness and feasibility of the present method.
\end{abstract}

\section{Introduction}

Recently, with the gradually thorough of the understanding of domestic and foreign scholars on the failure mechanism of mechanical components, and the preliminary grasp of random failure rule on mechanical components, on the basis of the reliability calculation model of mechanical components is established. At present, the uncertain parameters are random variables with theoretical probability distribution in this model ${ }^{[1-4]}$. But in practical engineering, there are some random variables which are not in conformity with the actual situation, for example, if the geometry size and strength limit of mechanical components are normally distributed, its value range is from the negative to the positive infinity, in fact, the geometry size and strength limit cannot be negative or infinite. In view of this, it is very necessary to establish a reasonable reliability calculation model of mechanical components on the condition of truncated normal distribution.

At present, research work on the reliability analysis of truncated distribution that there have been some reports. Literature [5] proposed to improve FORM algorithm for solving the reliability method problems with truncated distribution random variables. Literature [6] was combination of saddle-point approximation approach and FORM method, studies the reliability calculation method for random parameter of truncated distribution random variables.

In view of the above problems, this paper will carry out research work in the following aspects. First of all, according to the theoretical derivation for the first four moments of truncated normal distribution, than by random perturbation technique to get the first four moments of the state function; Secondly, the probability density function expression of state function are obtained by means of maximum entropy principle, on the basis of the mechanical components reliability of truncated normal distribution are obtained; Then, the optimization design theory melt into mechanical components reliability model of truncated normal distribution, and establish the optimization design model of mechanical components reliability with truncated normal distribution; Finally, through the study of reliability-based optimization design for the front axle, the calculation results show that the reliability of optimal design method is feasible and correct.

\section{The truncated normal distribution and moment}

The truncated normal distribution under the condition of $X \in(a, b)$ is usually recorded as 
$X \sim T N_{(a, b)}\left(\mu, \sigma^{2}\right)$. Where the design variables $Y=\frac{X-\mu}{\sigma}$ is given, the standard truncated normal distribution under the condition of $Y \in(\alpha, \beta)$ is recorded as $Y \sim T N_{(\alpha, \beta)}(0,1)$.

If $Y \sim T N_{(\alpha, \beta)}(0,1)$ is given, we obtain

$$
\mathrm{E}\left[(k+1) Y^{k}\right]-\mathrm{E}\left[Y^{k+2}\right]=\frac{\beta^{k+1} \phi(\beta)-\alpha^{k+1} \phi(\alpha)}{\Phi(\beta)-\Phi(\alpha)}, k=-1,0,1 \cdots
$$

The first four origin moment of the standard truncated normal random variable $Y$ by the recurrence formula (1) can be represented as follow

$$
\begin{aligned}
& \mathrm{E}(Y)=\frac{\phi(\alpha)-\phi(\beta)}{\Phi(\beta)-\Phi(\alpha)} \\
& \mathrm{E}\left(Y^{2}\right)=1+\frac{\alpha \phi(\alpha)-\beta \phi(\beta)}{\Phi(\beta)-\Phi(\alpha)} \\
& \mathrm{E}\left(Y^{3}\right)=2 \mathrm{E}(Y)-\frac{\beta^{2} \phi(\beta)-\alpha^{2} \phi(\alpha)}{\Phi(\beta)-\Phi(\alpha)} \\
& \mathrm{E}\left(Y^{4}\right)=3 \mathrm{E}\left(Y^{2}\right)-\frac{\beta^{3} \phi(\beta)-\alpha^{3} \phi(\alpha)}{\Phi(\beta)-\Phi(\alpha)}
\end{aligned}
$$

Where, $\alpha=\frac{a-\mu}{\sigma}, \beta=\frac{b-\mu}{\sigma}$.

According to the $Y=\frac{X-\mu}{\sigma}$, we can obtain the equation $X-\mu=\sigma Y$, and get the mean, variance, the third central moment and fourth central moment of truncated normal random variables $X$, then substituting equations $(2) \sim(5)$ into equations $(6) \sim(9)$, we obtain

$$
\begin{aligned}
\mu_{X} & =\mathrm{E}(X)=\mu+\sigma \mathrm{E}(Y) \\
\operatorname{Var}(X) & =\mathrm{E}(X-\mu)^{2}=\sigma^{2} \mathrm{E}\left(Y^{2}\right) \\
\mathrm{C} 3(X) & =\mathrm{E}(X-\mu)^{3}=\sigma^{3} \mathrm{E}\left(Y^{3}\right) \\
\mathrm{C} 4(X) & =\mathrm{E}(X-\mu)^{4}=\sigma^{4} \mathrm{E}\left(Y^{4}\right)
\end{aligned}
$$

\section{The maximum entropy principle}

Considering that the first origin moment of the standardized random variables $Y=\frac{Z-\mu}{\sigma}$ with the state function $Z=g(\boldsymbol{X})$ is used as the constraint condition, we obtain the maximum value of the information entropy ${ }^{[7]}$ :

$$
\left.\begin{array}{l}
\max H=-c \int_{-\infty}^{+\infty} f_{Y}(y) \ln f_{Y}(y) \mathrm{d} y \\
\text { st. } \mathrm{E}\left(Y^{i}\right)=\int_{-\infty}^{+\infty} y^{i} f_{Y}(y) \mathrm{d} y,(i=0,1, \cdots, m)
\end{array}\right\}
$$

Where, $c$ is constant and is greater than zero.

Using the method of Lagrange multiplier method (10), the maximum entropy probability density function can be gained

$$
f_{Y}(y)=\exp \left(-\sum_{i=0}^{m} b_{i} y^{i}\right)
$$

Where, $b_{0}, b_{1}, \cdots, b_{m}$ are undetermined coefficients.

The relationship between the center moment of $Z=g(X)$ and the origin moment of $Y$ can be represented as follow

$$
\mathrm{E}\left(Y^{i}\right)=\frac{\mathrm{E}\left[\left(Z-\mu_{\mathrm{g}}\right)^{i}\right]}{\sigma_{\mathrm{g}}^{i}}, i=0,1, \cdots, m
$$


Substituting equations (11) and (12) into equation (10), the integral equations can be obtained

$$
\int_{-\infty}^{+\infty} y^{i} \exp \left(-\sum_{i=0}^{m} b_{i} y^{i}\right) \mathrm{d} y=\mathrm{E}\left(Y^{i}\right), i=0,1, \cdots, m
$$

From the above formula, we can solve the coefficients $b_{0}, b_{1}, \cdots, b_{m}$, and obtain the probability density function expression of the standardized random variable $Y=\frac{Z-\mu}{\sigma}$ with the state function $Z=g(\boldsymbol{X})$.

The reliability of mechanical components can be expressed as

$$
R_{\mathrm{EM}}=\operatorname{Pr}(Z \geq 0)=\operatorname{Pr}\left(-\frac{\mu_{Z}}{\sigma_{Z}}\right)=\int_{-\frac{\mu_{Z}}{\sigma_{Z}}}^{+\infty} \exp \left(-\sum_{i=0}^{m} b_{i} y^{i}\right) \mathrm{d} y
$$

Where, $R_{\mathrm{EM}}$ denotes the reliability obtained by the maximum entropy principle. In engineering practice, the first four moments of the random variables are generally satisfied by the engineering design requests.

\section{Reliability-based Optimization Design of Truncated Normal Distributions}

The reliability-based optimization design model can be formulated in the following form

$$
\left.\begin{array}{lll}
\operatorname{minimize} & \mu_{\mathrm{f}} \\
\text { subject to } & R_{\mathrm{EM}} \geq R_{0} & \\
& q_{i}(\overline{\boldsymbol{X}}) \geq 0, \quad(i=1, \cdots, l) \\
& h_{j}(\overline{\boldsymbol{X}})=0, \quad(j=1, \cdots, m)
\end{array}\right\}
$$

Where, $\mu_{f}$ is the mean of the objective function, $R_{\mathrm{EM}}$ is maximum entropy reliability, $R_{0}$ is the reliability which be satisfied by the requests, $q_{i}(\overline{\boldsymbol{X}})$ and $h_{j}(\overline{\boldsymbol{X}})$ are respectively the inequality constraints and equality constraints.

\section{Numerical example}

The bending moment and torsion moment of the risk section are random variables with arbitrary distribution, the first four moments of the random variable are $(M)=\left(3.5192 \times 10^{6} \mathrm{~N} \cdot \mathrm{mm}\right.$, $\left.3.1961 \times 10^{5} \mathrm{~N} \cdot \mathrm{mm}, 3.6947 \times 10^{16}(\mathrm{~N} \cdot \mathrm{mm})^{3}, 5.5364 \times 10^{22}(\mathrm{~N} \cdot \mathrm{mm})^{4}\right),(T)=\left(3.0834 \times 10^{6} \mathrm{~N} \cdot \mathrm{mm}\right.$, $\left.2.5017 \times 10^{5} \mathrm{~N} \cdot \mathrm{mm}, 1.806 \times 10^{16}(\mathrm{~N} \cdot \mathrm{mm})^{3}, 2.135 \times 10^{22}(\mathrm{~N} \cdot \mathrm{mm})^{4}\right)$ respectively; The strength of material is the truncated normal random variables, the mean value and standard deviation of them were respectively $(r)=(667,25.3) \mathrm{MPa}$, left and right truncation points are 606 and 718 . Supposing the required reliability $R_{0}=0.99$, we could try to use the reliability-based optimization design method to confirm the geometry size of the front axle: $a, t, h$ and $b$.

At first, established the objective function:

To require the lightest weight of the front axle, which is that we must find the smallest front axle section area $A-f(x)$ :

where the design variables are $\boldsymbol{x}=\left[\begin{array}{llll}x_{1} & x_{2} & x_{3} & x_{4}\end{array}\right]^{T}=\left[\begin{array}{lll}a & t & h\end{array}\right]^{T}$.

$$
f(x)=x_{1}\left(x_{3}-2 x_{2}\right)+2 x_{4} x_{2}
$$

Secondly, establishing constraint condition:

$$
\begin{gathered}
R_{\mathrm{EM}} \geq R_{0} \\
x_{3}-2 x_{2} \geq 0
\end{gathered}
$$

Last, optimal solution:

The initial values, $a=12 \mathrm{~mm}, t=14 \mathrm{~mm}, h=85 \mathrm{~mm}, b=65 \mathrm{~mm}$ are given, and the solution for $a$, $t, h, b$ of optimization is

$$
a=10.8857 \mathrm{~mm}, t=12.8857 \mathrm{~mm}, h=80 \mathrm{~mm}, b=60 \mathrm{~mm}
$$


According to the results that computed by the reliability-based optimization design method, the reliability and relative error of reliability of the front axle are respectively:

$$
\begin{gathered}
R_{\mathrm{EM}}=0.99, R_{\mathrm{FM}}=0.9854, R_{\mathrm{MCS}}=0.9918, \\
\varepsilon_{\mathrm{R} 1}=\left|\frac{R_{\mathrm{EM}}-R_{\mathrm{MCS}}}{R_{\mathrm{MCS}}}\right|=\left|\frac{0.99-0.9918}{0.9918}\right|=0.0018, \\
\varepsilon_{\mathrm{R} 2}=\left|\frac{R_{\mathrm{FM}}-R_{\mathrm{MCS}}}{R_{\mathrm{MCS}}}\right|=\left|\frac{0.9854-0.9918}{0.9918}\right|=0.0064
\end{gathered}
$$

where, $R_{\mathrm{EM}}$ is the reliability that computed by the principle of maximum entropy, $\varepsilon_{\mathrm{R} 1}$ is the relative error of the reliability $R_{1}, R_{\mathrm{FM}}$ is the reliability that computed by four moment method, $\varepsilon_{\mathrm{R} 2}$ is the relative error of the reliability $R_{2}, R_{\mathrm{MCS}}$ is the reliability that computed by Monte Carlo simulation (MCS).

\section{Conclusion}

Based on the theory of truncated distribution, random perturbation technique, the maximum entropy principle and the reliability-based optimization design method, this paper studies the reliability-based optimization design problems of mechanical components with truncated normal distribution.

The truncated normal distribution is seen as a new distribution, on the condition of known first second moments of original normal distribution and the truncation points, you can obtain the first four moments, the reliability-based optimization design is approximately expressed by the first four moments using the maximum entropy method. The probability density function of state function obtained by the maximum entropy principle can be well approximation to the real distribution, so reliability by this method has higher precision.

\section{References}

[1] Y. M. Zhang: Chinese Journal of Mechanical Engineering Vol. 14 (2010), p.167

[2] DUBOURG V, SUDRET B, BOURINET J M: Structural and Multidisciplinary Optimization Vol. 5(2011), p.673

[3] Y. F. Tang, J. Q. Chen, J. H. Wei: ASME Journal of Mechanical Design Vol. 1(2012), p.014502

[4] NOH Y, CHOI K K, DU L: Structural and Multidisciplinary Optimization Vol. 1(2009), p.1

[5] MELCHERS R E, AHAMMED M, MIDDLETON C: Structural Safety Vol. 25(2003), p.305

[6] DU X P, HU Z: ASME Journal of Mechanical Design Vol. 9(2012), p.091005

[7] KESAVAN H K, KAPUR J N: Entropy Optimization Principles with Applications, Academic Press: New York (1992) 